\title{
Bioprótese Valvar Porcina: Um Legado de Mario Vrandecic
}

\section{Porcine Valve Bioprosthesis: a Legacy from Mario Vrandecic}

Erika Correa Vrandecic, ${ }^{1}$ Ektor Correa Vrandecic, ${ }^{1}$ Bayard Contijo-Filho, ${ }^{1}$ Rossana Dall'Orto Elias, ${ }^{1}$ Braulio Roberto Gonçalves Marinho Couto, ${ }^{1}$ Marcus Vinicius Bolivar Malachias ${ }^{1,2}{ }^{-}$

Biocor Instituto, ${ }^{1}$ Nova Lima, MG - Brasil

Faculdade de Ciências Médicas de Minas Gerais, ${ }^{2}$ Belo Horizonte, MG - Brasil

A doença valvar cardíaca ocupa atualmente os holofotes da medicina cardiovascular em face dos recentes avanços das técnicas de imagens e emergentes possibilidades terapêuticas, atraindo a atenção de médicos, pesquisadores, fabricantes de dispositivos e investidores. ${ }^{1}$ O Brasil ocupa um lugar de destaque internacional na história e no desenvolvimento tecnológico de substitutos valvares utilizados no tratamento dessa enfermidade.

O primeiro implante mundial de uma bioprótese valvar suína, comercialmente disponível, aconteceu em outubro de $1968 .{ }^{2}$ Cerca de meio século depois, em setembro de 2019, faleceu o médico e cientista Mario Vrandecic, criador da única bioprótese cardíaca de tecido porcino produzida no Brasil e aprovada na agência norte-americana Food and Drug Adminstration (FDA), há décadas globalmente utilizada no tratamento da doença cardíaca valvar.

Nesse artigo, destacamos a história do seu criador e as evidências de efetividade e segurança da bioprótese valvar Biocor, hoje denominada St. Jude Medical Biocor (St. Jude Medical, Inc., St Paul, MN). ${ }^{3-16}$

Mario Vrandecic, de ascendência croata e nascido na Bolívia, cursou Medicina na Faculdade de Medicina da Universidade Federal de Minas Gerais (FM/UFMG). Especializou-se em cirurgia geral e cardiovascular nos Estados Unidos da América (EUA), onde serviu ao exército americano como cirurgião, inclusive durante a guerra do Vietnã. Retornou definitivamente ao Brasil em 1976 e passou a atuar como professor da FM/UFMG e cirurgião cardiovascular na Santa Casa de Belo Horizonte, entre outros hospitais.

Tendo realizado pesquisas com tecidos biológicos durante a sua residência nos EUA, em 1981 criou a Biocor Indústria, empresa na qual desenvolveu uma bioprótese valvar cardíaca de tecido porcino, entre outras patentes. Inicialmente usada no Brasil, na América Central e na Ásia, a bioprótese logo obteve o CE Marking, passando a ser utilizada na Europa e, posteriormente, com a aprovação pelo FDA, também nos EUA. Recebeu homenagens de várias sociedades científicas e de entidades da área de inovação nacionais e internacionais. Em 1997, a Biocor Indústria foi vendida à empresa norte-americana

\section{Palavras-chave}

Doença das Valvas Cardíacas/cirurgia; Implante de Prótese de Valva Cardíaca; Biopróteses/tendências; Mario Vrandecic.

Correspondência: Marcus Vinicius Bolivar Malachias •

Biocor Instituto - Rua Mario Vrandecic, 250. CEP 34006-059, Bairro Vila da Serra, Nova Lima, MG - Brasil

E-mail: mbolivar@cardiol.br

Artigo recebido em 15/10/2020, revisado em 18/11/2020, aceito em 18/11/2020

DOI: https://doi.org/10.36660/abc.20201110
St. Jude Medical, sendo posteriormente incorporada no ano de 2016 à Abbott Laboratories.

Após quase 40 anos de uso clínico, as evidências de seguimentos de curto, médio e longo prazos demonstram efetividade, durabilidade e segurança da referida bioprótese valvar em séries nacionais e de outros países (Tabela 1). ${ }^{3-16}$ Em um dos seguimentos mais longos, Mykén e Bech-Hansen, avaliaram 1.712 pacientes que receberam a bioprótese Biocor porcina no Sahlgrenska University Hospital, em Gotemburgo, na Suécia, demonstrando uma sobrevida livre de mortalidade devido à falência valvar após 20 anos de $84,3 \% \pm 6,9 \%$ e 88,0\% $\pm 4,0 \%$, para implantes em posições aórtica e mitral, respectivamente ${ }^{16}$ (Tabela 1).

Mario Vrandecic fundou ainda, em 1985, o Biocor Instituto, localizado em Nova Lima, região metropolitana de Belo Horizonte (MG). Inicialmente dedicado às doenças cardiovasculares, o hospital logo evoluiu para ser um importante centro de Medicina de alta complexidade. O hospital tem sido o responsável pela especialização e atuação de muitas gerações de cardiologistas, cirurgiões cardíacos, médicos de diversas especialidades e outros profissionais de saúde, além de ser referência em assistência de qualidade à população do estado, com importantes certificações nacionais e internacionais. A gestão de Mario Vrandecic foi pautada na ética, na geração de confiança, na qualificação de pessoas e na educação continuada. O seu legado simboliza um exemplo de humanismo e dedicação à Medicina, um marco de inovação em ciência cardiovascular e uma prova da potencialidade biotecnológica do país.

\section{Contribuição dos autores}

Concepção e desenho da pesquisa, Análise e interpretação dos dados e Revisão crítica do manuscrito quanto ao conteúdo intelectual: Vrandecic EC, Vrandecic EC, Gontijo-Filho B, Elias RD, Couto BRGM, Malachias MVB; Obtenção de dados: Vrandecic EC, Vrandecic EC, Gontijo-Filho B; Análise estatística e Redação do manuscrito: Vrandecic EC, Couto BRGM, Malachias MVB.

\section{Potencial conflito de interesses}

Os autores declaram não haver conflito de interesses pertinentes.

\section{Fontes de financiamento}

O presente estudo não contou com fontes de financiamento externas.

\section{Vinculação acadêmica}

Não há vinculação deste estudo a programas de pós-graduação. 


\section{Carta Científica}

\begin{tabular}{|c|c|c|c|c|c|}
\hline $\begin{array}{l}\text { Autorl } \\
\text { referência }\end{array}$ & Período de acompanhamento & $\begin{array}{l}\text { Posição da } \\
\text { bioprótese }\end{array}$ & $\begin{array}{l}\text { Tamanho da } \\
\text { amostra (n) }\end{array}$ & Desfecho & Resultado observado \\
\hline \multirow{6}{*}{ Vrandecic ${ }^{3,4}$} & \multirow{6}{*}{$\begin{array}{c}\text { Março 1981- } \\
\text { março 1988 } \\
\text { (48 [1 a 84] meses) }\end{array}$} & \multirow{2}{*}{$\begin{array}{l}\text { Aórtica + } \\
\text { Mitral }\end{array}$} & \multirow{2}{*}{1.713} & Mortalidade hospitalar & $6,1 \%$ \\
\hline & & & & Sobrevida após 7 anos & $97,1 \%$ \\
\hline & & \multirow[b]{2}{*}{ Aórtica } & \multirow[b]{2}{*}{385} & Complicações tardias & $13,2 \%$ \\
\hline & & & & $\begin{array}{l}\text { Sobrevida livre de falência } \\
\text { valvar após } 7 \text { anos }\end{array}$ & $96,9 \%$ \\
\hline & & \multirow{2}{*}{ Mitral } & \multirow{2}{*}{716} & Complicações tardias & $14,2 \%$ \\
\hline & & & & Sobrevida após 7 anos & $95,2 \%$ \\
\hline Gontijo-Filho ${ }^{5}$ & $\begin{array}{c}\text { Maio } 1990- \\
\text { março } 1992 \\
\text { (9 }[1 \text { a 22] meses) }\end{array}$ & Aórtica & 81 & Mortalidade hospitalar & $4,9 \%$ \\
\hline Gontijo-Filho ${ }^{6}$ & $\begin{array}{l}\text { Junho } 1990- \\
\text { janeiro } 1993\end{array}$ & $\begin{array}{c}\text { Aórtica } \\
\text { stentless } \\
\text { em } \\
\text { alterações do } \\
\text { anel aórtico }\end{array}$ & 16 & Mortalidade hospitalar & $6,3 \%$ \\
\hline \multirow{2}{*}{ Vrandecic $^{7}$} & \multirow{2}{*}{$\begin{array}{c}\text { Março } 1992- \\
\quad \text { março } 1993 \\
\text { (6 [1 a 12] meses) }\end{array}$} & \multirow{2}{*}{ Mitral } & \multirow{2}{*}{38} & Mortalidade hospitalar & $0 \%$ \\
\hline & & & & Retroca valvar & $3,8 \%$ \\
\hline \multirow{2}{*}{ Vrandecic $^{8}$} & \multirow{2}{*}{ Maio 1990 -dezembro 1993} & \multirow{2}{*}{$\begin{array}{l}\text { Áortica } \\
\text { stentless }\end{array}$} & \multirow{2}{*}{120} & Mortalidade hospitalar & $5 \%$ \\
\hline & & & & Retroca valvar & $4 \%$ \\
\hline \multirow{2}{*}{ Vrandecic $^{9}$} & \multirow{2}{*}{ (14 [1 a 26] meses) } & \multirow{2}{*}{$\begin{array}{c}\text { Mitral } \\
\text { stentless }\end{array}$} & \multirow{2}{*}{85} & Mortalidade hospitalar & $0 \%$ \\
\hline & & & & Retroca valvar & $6 \%$ \\
\hline \multirow{2}{*}{ Vrandecic $^{10}$} & \multirow{2}{*}{$\begin{array}{l}\text { Março } 1992 \text { - dezembro } 1995 \\
\quad(26 \text { [3 a 45] meses) }\end{array}$} & \multirow{2}{*}{$\begin{array}{c}\text { Mitral } \\
\text { stentless }\end{array}$} & \multirow{2}{*}{108} & Mortalidade hospitalar & $6,5 \%$ \\
\hline & & & & Retroca valvar & $12,5 \%$ \\
\hline \multirow{2}{*}{ Vrandecic ${ }^{11}$} & \multirow{2}{*}{$\begin{array}{c}\text { Março } 1992- \\
\text { agosto } 1996 \\
\text { (29 [2 a 54] meses) }\end{array}$} & \multirow{2}{*}{$\begin{array}{c}\text { Mitral } \\
\text { stentless }\end{array}$} & \multirow{2}{*}{120} & Mortalidade hospitalar & $6,5 \%$ \\
\hline & & & & Retroca valvar & $14,3 \%$ \\
\hline Vrandecic $^{12}$ & $\begin{array}{c}\text { Janeiro } 1990- \\
\text { junho } 1999 \\
\text { (54 [3 a 114] meses) }\end{array}$ & $\begin{array}{l}\text { Aórtica } \\
\text { stentless vs } \\
\text { stented }\end{array}$ & 407 & Sobrevida em 8 anos & $\begin{array}{c}71,8 \% \pm 0,7 \% \text { (stentless) } \\
\text { vs } \\
62,9 \% \pm 13,4 \% \text { (stented) }\end{array}$ \\
\hline \multirow{9}{*}{ Kirali $^{13}$} & \multirow{9}{*}{$\begin{array}{l}\text { Janeiro } 1985- \\
\text { junho 1999 } \\
\text { (10 [1 a 15] anos) }\end{array}$} & \multirow{9}{*}{ Mitral } & \multirow{9}{*}{158} & Mortalidade em 30 dias & $4,4 \%$ \\
\hline & & & & Retroca valvar & $14 \%$ \\
\hline & & & & Sobrevida livre em 5 anos & $83,7 \% \pm 3 \%$ \\
\hline & & & & Sobrevida em 13 anos & $77,8 \% \pm 3,4 \%$ \\
\hline & & & & $\begin{array}{l}\text { Sobrevida livre de falência valvar } \\
\text { após } 5 \text { anos }\end{array}$ & $95,5 \% \pm 1.8 \%$ \\
\hline & & & & $\begin{array}{l}\text { Sobrevida livre de falência valvar } \\
\text { após } 13 \text { anos }\end{array}$ & $64,8 \% \pm 5,3 \%$ \\
\hline & & & & $\begin{array}{l}\text { Sobrevida livre de reoperação, } \\
\text { devido falência valvar após } 5 \text { anos }\end{array}$ & $98,4 \% \pm 1,1 \%$ \\
\hline & & & & $\begin{array}{c}\text { Sobrevida livre de reoperação, } \\
\text { devido falência valvar após } 10 \\
\text { anos }\end{array}$ & $89,2 \% \pm 2,9 \%$ \\
\hline & & & & $\begin{array}{c}\text { Sobrevida livre de reoperação, } \\
\text { devido falência valvar após } 14 \\
\text { anos }\end{array}$ & $76,8 \% \pm 7,9 \%$ \\
\hline
\end{tabular}


Carta Científica

\begin{tabular}{|c|c|c|c|c|c|}
\hline Continuação & & & & & \\
\hline \multirow{3}{*}{ Pomerantzeff ${ }^{14}$} & \multirow{3}{*}{$\begin{array}{l}\text { Março } 1983- \\
\text { dezembro } 2000\end{array}$} & \multirow{3}{*}{ Mitral } & \multirow{3}{*}{546} & Mortalidade hospitalar & $9,5 \%$ \\
\hline & & & & Sobrevida em 15 anos & $45 \% \pm 15,8 \%$ \\
\hline & & & & $\begin{array}{c}\text { Sobrevida livre de reoperação, } \\
\text { devido falência valvar após } 15 \\
\text { anos }\end{array}$ & $33,9 \% \pm 10,4 \%$ \\
\hline \multirow{13}{*}{ Eichinger $^{15}$} & \multirow{13}{*}{$\begin{array}{l}\text { Janeiro } 1985 \text {-dezembro } 2006 \\
\text { (8 [1 a 21] anos) }\end{array}$} & \multirow{13}{*}{ Aórtica } & \multirow{13}{*}{455} & Mortalidade em 30 dias & $5.3 \%$ \\
\hline & & & & Sobrevida em 5 anos & $74,7 \% \pm 2,0 \%$ \\
\hline & & & & Sobrevida em 10 anos & $44,9 \% \pm 2,4 \%$ \\
\hline & & & & Sobrevida em 15 anos & $20,9 \% \pm 2,5 \%$ \\
\hline & & & & Sobrevida em 20 anos & $9,4 \% \pm 2,8 \%$ \\
\hline & & & & $\begin{array}{l}\text { Sobrevida livre de falência valvar } \\
\text { após } 5 \text { anos }\end{array}$ & $97,5 \% \pm 0,8 \%$ \\
\hline & & & & $\begin{array}{l}\text { Sobrevida livre de falência valvar } \\
\text { após } 10 \text { anos }\end{array}$ & $93,1 \% \pm 1,7 \%$ \\
\hline & & & & $\begin{array}{c}\text { Sobrevida livre de falência valvar } \\
\text { após } 15 \text { anos }\end{array}$ & $88,4 \% \pm 3,5 \%$ \\
\hline & & & & $\begin{array}{c}\text { Sobrevida livre de falência valvar } \\
\text { após } 20 \text { anos }\end{array}$ & $70,3 \% \pm 10,9 \%$ \\
\hline & & & & $\begin{array}{l}\text { Sobrevida livre de reoperação, } \\
\text { devido falência valvar após } 5 \text { anos }\end{array}$ & $95,9 \% \pm 1 \%$ \\
\hline & & & & $\begin{array}{c}\text { Sobrevida livre de reoperação, } \\
\text { devido falência valvar após } 10 \\
\text { anos }\end{array}$ & $91,9 \% \pm 1,6 \%$ \\
\hline & & & & $\begin{array}{c}\text { Sobrevida livre de reoperação, } \\
\text { devido falência valvar após } 15 \\
\text { anos }\end{array}$ & $90,6 \% \pm 2,1 \%$ \\
\hline & & & & $\begin{array}{c}\text { Sobrevida livre de reoperação, } \\
\text { devido falência valvar após } 20 \\
\text { anos }\end{array}$ & $86,5 \% \pm 4,5 \%$ \\
\hline \multirow{8}{*}{ Mykén ${ }^{16}$} & \multirow{8}{*}{$\begin{array}{l}\text { Janeiro } 1983- \\
\text { janeiro } 2003 \\
\text { [média } 6 \text { anos] }\end{array}$} & \multirow{4}{*}{ Aórtica } & \multirow{4}{*}{1.518} & Mortalidade hospitalar & $5,1 \%$ \\
\hline & & & & Incidência de reoperação & $\begin{array}{c}0,9 \% / \\
\text { paciente-ano }\end{array}$ \\
\hline & & & & $\begin{array}{c}\text { Sobrevida livre de mortalidade, } \\
\text { devido falência valvar após } 20 \\
\text { anos }\end{array}$ & $84,3 \% \pm 6.9 \%$ \\
\hline & & & & $\begin{array}{l}\text { Sobrevida livre de reoperação, } \\
\text { devido falência valvar após } 20 \\
\text { anos }\end{array}$ & $61,1 \% \pm 8,5 \%$ \\
\hline & & \multirow{4}{*}{ Mitral } & \multirow{4}{*}{194} & Mortalidade hospitalar & $12,9 \%$ \\
\hline & & & & Incidência de reoperação & $\begin{array}{c}0,9 \% / \\
\text { paciente-ano }\end{array}$ \\
\hline & & & & $\begin{array}{c}\text { Sobrevida livre de mortalidade, } \\
\text { devido falência valvar após } 20 \\
\text { anos }\end{array}$ & $88,0 \% \pm 4,0 \%$ \\
\hline & & & & $\begin{array}{l}\text { Sobrevida livre de reoperação, } \\
\text { devido falência valvar após } 20 \text { anos }\end{array}$ & $79,3 \% \pm 6,0 \%$ \\
\hline
\end{tabular}

*Dados de 14 publicações que avaliaram os desfechos de curto, médio e longo prazos das biopróteses porcinas Biocor, publicados entre 1988 e $2008 .{ }^{3-16}$ 


\section{Carta Científica}

\section{Referências}

1. Binder RK, Dweck M, Prendergast B. The year in cardiology: valvular heart disease. Eur Heart J. 2020; 41(8): 912-20.

2. Bortolotti U, Milano AD, Valente M, Thiene G. The Stented Porcine Bioprosthesis: a 50-year journey through hopes and realities. Ann Thorac Surg. 2019; 108(1):304-8.

3. Vrandecic MO, Filho BG, Silva JAP,Fantini FA, Barbosa IT, São Jose MC, et al. Clinical results with the Biocor porcine bioprosthesis. J Cardiovasc Surg (Torino). 1991; 32(6): 807-13.

4. Vrandecic MO, Filho BG, Silva JAP,Radegran KI, Silva JAP < Fantini FA, et al. Estudo multicêntrico dos resultados das trocas valvares com o uso da bioprótese Biocor no Estado de Minas Gerais. Rev Bras Cir Cardiovasc. 1988; 3(3): 159-68.

5. Gontijo Filho B, Vrandecic MO, Morea M, Morea M, Nova bioprótese aórtica sem suporte: resultados clínicos. Rev Bras Cir Cardiovasc. 1992; 7(3): 208-14.

6. Gontijo Filho B, Vrandecic MO, Fantini FA, Barbosa JT, Avelar SS, et al. Implante de bioprótese aórtica "stentless" em pacientes com alterações do anel aórtico. Rev Bras Cir Cardiovasc. 1993; 8(2): 118-24.

7. Vrandecic MO, Filho BG, Fantini FA, Barbosa JT, Silva JAP, Barbosa JT, Gutierrez C, et al. Transplante de valva mitral heteróloga: nova alternativa cirúrgica: estudo clínico inicial. Rev Bras Cir Cardiovasc. 1993; 8(2): 83-90.

8. Vrandecic MO, Filho BG, Fantini FA, Oliveira OC, Martins Jr IC, Bioprótese aórtica porcina "stentless": acompanhamento clínico a médio prazo. Rev Bras Cir Cardiovasc. 1994; 9(1): 60-3.
9. Vrandecic MOP, Fantini FA, Gontijo BF,Oliveira OC, Martins Jr JC. Surgical technique of implanting the stentless porcine mitral valve. Ann Thorac Surg. 1995; 60 (2 Suppl): S439-S442.

10. Vrandecic MO, Filho BG, Fantini FA, Martins MH, Avelar SS, Vandrecic E. Valva mitral heteróloga sem suporte: resultados clínicos a médio prazo. Rev Bras Cir Cardiovasc. 1996; 11(3): 148-54.

11. Vrandecic MO, Gontijo B, Fantini FA, Martins I, Oliveira MH, Avelar SS, et al. Porcine mitral stentless valve mid-term clinical results. Eur J Cardiothorac Surg. 1997; 12(1): 56-62.

12. Vrandecic M, Fantini FA, Filho BG, Filho BG, Oliveira OC, Costa Jr IM, et al. Retrospective clinical analysis of stented vs stentless porcine aortic bioprostheses. Eur J Cardiothorac Surg. 2000; 18: 46-53.

13. Kirali K, Güler M, Tuncer A, Daglar B, Ipek G, Isik O, et al. Fifteen-year clinical experience with the biocor porcine bioprostheses in the mitral position. Ann Thorac Surg. 2001; 71(3): 811-5.

14. Pomerantzeff PM, Brandão CM, Albuquerque JM, Stolf NA, Grinberg M, Oliveira AS. Long-term follow up of the Biocor porcine bioprosthesis in the mitral position. J Heart Valve Dis. 2006; 15(6): 763-7.

15. Eichinger WB, Hettich IM, Ruzicka DJ, Holper K, Schricker C, Bleiziffer S, et al. Twenty-year experience with the St. Jude medical Biocor bioprosthesis in the aortic position. Ann Thorac Surg. 2008; 86(4):1204-10.

16. Mykén PS, Bech-Hansen O. A 20-year experience of 1712 patients with the Biocor porcine bioprosthesis. J Thorac Cardiovasc Surg. 2009; 137(1): 76-81. 